\title{
On the use of dynamic state estimation for the optimal control of power system
}

\author{
Alexander Domyshev, ${ }^{1, *}$, and Alexander Tikhonov ${ }^{2}$ \\ ${ }^{1}$ Melentiev Energy Systems Institute of SB RAS, Irkutsk,Russia \\ ${ }^{2}$ RUSAL Engineering and Technological Center, LTD, Irkutsk, Russia
}

\begin{abstract}
Investigations of the application of classical methods of dynamic state estimation on the data of a real power system for the purposes of optimal control have been carried out. A modern feature of state estimation for a large power system is that data from the SCADA system are fed to the calculation subsystem with a fairly small frequency. It is shown that the use of classical methods of dynamic state estimation for such problems is limited. The study was carried out using the ergodic theory of a dynamical system.
\end{abstract}

\section{Introduction}

The diversification of the energy sector in the Russian Federation and the subsequent forced digitalization were the catalyst for the creation of an intelligent energy system in Russia. Such an electric power system (EPS) contains a significant number of elements, the mode of which is stochastic in time. This is due to the large number of local control devices, the algorithm of which is not defined at the power system level. Along with this, in distribution networks, the problem of optimal control is become even more complicated due to the small number of measuring devices and significant uncertainty of measurements. To solve the problems of operational and emergency control, a mathematical model of the current state of electric grid is used.

At the same time, for the problem of optimal control, and especially for the problem of automatic optimal control, a reliably functioning state estimation algorithm [1] is required that works without human intervention.

In static state estimation, one uses the relationships between physical parameters of the single steady state, but there is additional information about the change in these parameters over time. This information ca $\mathrm{n}$ be used by applying dynamic state estimation algorithms. At the same time, the practical application of dynamic state estimation algorithms for optimal control purposes encounters computational difficulties. In this paper, an attempt is made to investigate the possibility of using existing dynamic state estimation. This information can be used by applying dynamic state estimation algorithms on a model of a sufficiently large power system using real telemetry received from a SCADA system.

\section{State estimation problem statement}

In a static formulation, the assessment of the EPS state is the calculation of the parameters of the state, carried out on the base of SCADA measurements $\bar{y}$.

$$
\bar{y}=\left[P_{i}, Q_{i}, P_{i j}, Q_{i j}, U_{i}, I_{i}, I_{i j}\right]
$$

The measurements vector includes: modules of nodal voltages $U_{i}$, generation of active $P_{i}$ and reactive power $Q_{i}$ in nodes, power flows of active $P_{i j}$ and reactive power $Q_{i j}$ through overhead lines and transformers, less often currents at the ends of overhead lines $\mathrm{I}_{\mathrm{ij}}$ and nodal currents $\mathrm{I}_{\mathrm{i}}$, some integral characteristics of the mode. To obtain nodal injections, in addition to measurements of loads and generation power, pseudo-measurements are used.

The task of state estimation is to find such estimates of the measured parameters $y(x)$ that are closest to the measured values $\bar{y}$. The sum of the weighted squares of the deviations of estimates from measurements is most often used as a criterion for proximity [2].

$$
J=[\bar{y}-y(x)]^{T} R_{v}^{-1}[\bar{y}-y(x)]
$$

where $R_{v}^{-1}$ is a diagonal matrix of weight coefficients whose elements are inverse to the variances of measurements characterizing their accuracy.

Estimates must satisfy the electrical circuit equations:

$$
w(y(x), x)=0
$$

The result of state estimation is the state vector

$$
\hat{x}=\left[\widehat{U}_{1}, \widehat{U}_{2}, \ldots, \widehat{U}_{n}, \hat{\delta}_{1}, \hat{\delta}_{2}, \ldots, \hat{\delta}_{n}\right]^{T}
$$

This vector contains the estimated voltages and its angles for each node. $\mathrm{n}$ - the total number of nodes in the model of the electrical grid.

Within the framework of the problem of dynamic state estimation, the system is usually considered to be Markov. The change in the state vector of system (1) is considered in the form of the following Markov process: 


$$
x_{i}=F\left(x_{i-1}\right)+\left[\widehat{U}_{1}, \widehat{U}_{2}, \ldots, \widehat{U}_{n}, \hat{\delta}_{1}, \hat{\delta}_{2}, \ldots, \hat{\delta}_{n}\right]^{T}
$$

To predict weakly variable components of the system state vector $x$ for a short period of time, a dynamic state estimation is used based on a modification of the Kalman filter.

The Kalman filter is a classic dynamic state estimation method. The essence of the Kalman filter is as follows. Suppose there is a time-varying parameter. The law of its change is known only with a certain error, so that:

$$
x_{i+1}=x_{i}+u\left(x_{i}\right)+\xi_{i},
$$

where $u($.$) is the assumed law of variation of x, \xi_{i}$ is an uncertain value. Also, at each time step (starting from the first), there are actual measurements of the predicted parameter $z_{i+1}=x_{i+1}+\eta_{i+1}$, containing the measurement error.

The idea behind the Kalman filter is that to get the best approximation to the proper value of $x_{i+1}$, one need to choose a compromise between measuring $z_{i+1}$ and inaccurate prediction $x_{i}+u\left(x_{i}\right)$. The measurement is assigned a weight of $K_{i}$, and the predicted value is assigned $1-K_{i}$, respectively. The Kalman coefficient changes at each iteration and is found by iteratively minimizing the squared prediction error

$$
E\left(e_{i+1}^{2}\right)=\frac{\sigma_{\eta}^{2}\left(E\left(e_{i}^{2}\right)+\sigma_{\xi}^{2}\right)}{E\left(e_{i}^{2}\right)+\sigma_{\xi}^{2}+\sigma_{\eta}^{2}}
$$

and the error-minimizing value of the Kalman coefficient on the next iteration

$$
K_{i+1}=\frac{E\left(e_{i+1}^{2}\right)}{\sigma_{\eta}^{2}},
$$

where $\sigma_{\eta}^{2}$ is the variance of the measurement error, and $\sigma_{\xi}^{2}$ is the variance of the model error.

For the nonlinear model $u\left(x_{i}\right)$, an extended Kalman filter is used [3,4] or faster methods that approximate nonlinearity, such as the Sigma-point Kalman filter (Unscented Kalman Filter), based on the same-name Unscented transformation.

A modern feature of state estimation for a large power system is that data from the SCADA system is fed to the calculation subsystem with a fairly small frequency. In the power system under consideration, the snapshots of measurements is formed at the 30-minute boundary. If there is WAMS, you can get consistent data much more often. In this case, the status evaluation period can be shortened to 10 seconds. However, for the purposes of automatic optimal control, when the problems of emergency management are not considered, the formation of a snapshots at the 30-minute boundary and, accordingly, the state estimation may be quite sufficient.

The use of classical methods of dynamic state estimation [5] on 30-minute snapshots of measurements obtained from SCADA, as shown by calculations, was not effective. Indeed, the change in load showed a chaotic nature. Fig. 1 shows the change over two days of the measured active load power and its estimates using a static state estimation algorithm. On the ordinate axis, the sequential number of the snapshot (half-hour) is deferred. Changes in values are given for a single node, but the nature of changes is similar for most load nodes in the network.

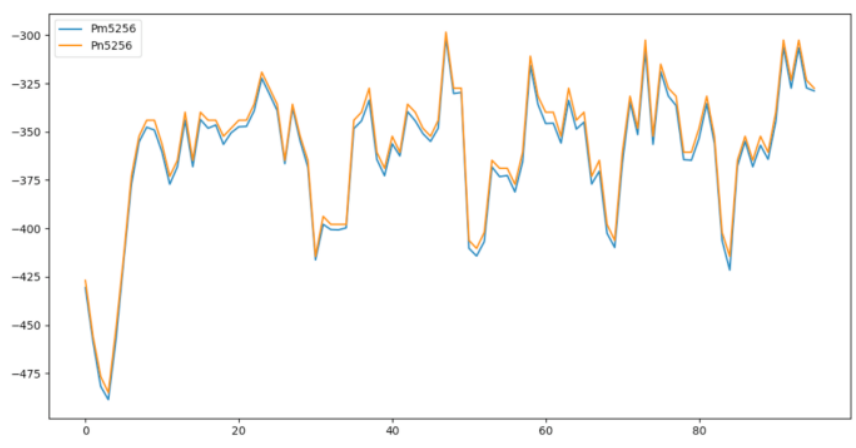

Fig. 1. Measured and estimated load in one of the grid nodes.

Similar chaotic behavior is observed in voltage and power flow measurements (Fig. 2, Fig. 3).

As a result of applying the Kalman filter with a linear or moving average model, we get a mode with a greater error than with static state estimation. In this case, there is either a delay and a roughen of the state (Fig. 4), or in some cases there is a outage of the computational stability of the algorithm. This behavior is explained by the fact that the error of the model $\xi_{i}$ significantly exceeds the measurement error $\eta_{i+1}$.

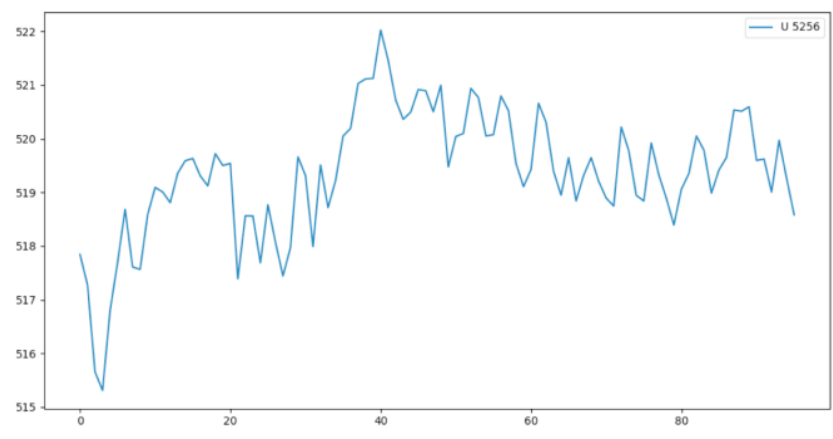

Fig. 2. Measured voltage.

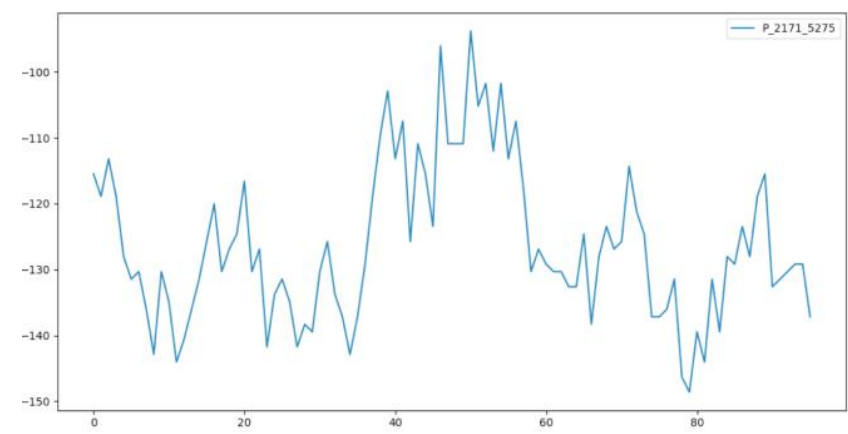

Fig. 3. Measured power flow.

In order to understand the possibility of creating an adequate model for predicting the process of changing sates parameters over time, the ergodic theory $[6,7]$ of dynamic chaos was applied. The process of changing states was considered as a dynamic system with an unknown control law. 


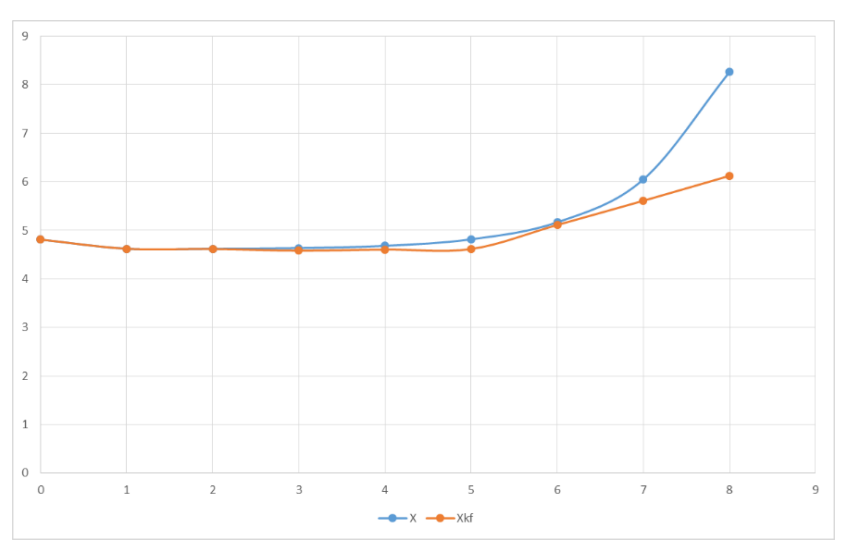

Fig. 4. Lag of the value filtered by Sigma-point Kalman filter. $\mathrm{X}$ - measured value, $\mathrm{Xkf}$ - filtered value.

To study the behavior of a system in the vicinity of an arbitrary trajectory, we use Lyapunov exponents that characterize the degree of stretching and compression in the phase space of the system's motion (changes in its parameters) along stable and unstable directions (5).

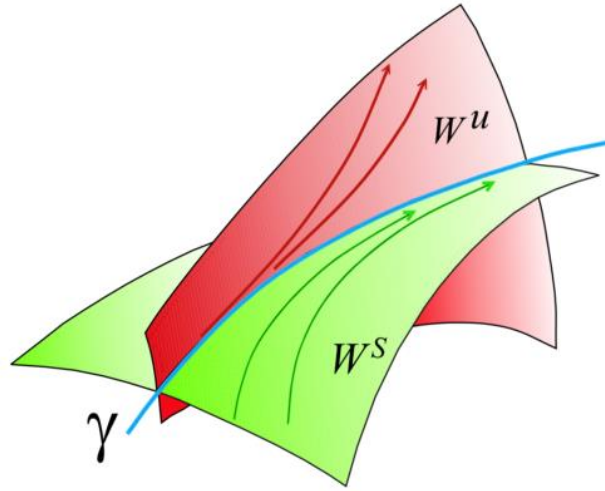

Fig. 5. The trajectory of the system is $\gamma . W^{S}$ is a stable manifold, $W^{U}$ is an unstable manifold of the system's trajectories (the figure is taken from [7]).

Let the dynamics of the system be given by a system of differential equations:

$$
\dot{x}=f(x(t), c),
$$

where $x$ is a vector of dynamic variables that depend on time $t$, and $c$ is a set of non-changing parameters. Consider the typical phase trajectory $x(t)$ of the system (2) and the trajectory close to it:

$$
x_{1}(t)=x(t)+\xi(t) .
$$

The function that defines Lyapunov exponents is written as:

$$
\Lambda(\xi)=\lim _{t \rightarrow \infty} \frac{1}{t} \ln \frac{|\xi(t)|}{|\xi(0)|}
$$

For $\xi(0) \rightarrow 0$, the values of function (3) are a vector with a dimension equal to the dimension of the phase space $n$ :

$$
\Lambda(\xi)=\left\{\lambda_{1}, \lambda_{2}, \ldots, \lambda_{n}\right\}
$$

If $\Lambda(\xi)$ does not contain positive values, then there is no chaotic component and the evolution of the system is completely predictable.
Obtaining the law of changing of the state parameters in time in the form (2) is impossible due to the complexity of the system under consideration. Therefore, it is necessary to apply the method of reconstructing a dynamic system from the available measurements using Takens' theorem. This theorem substantiates the possibility of reconstructing a strange attractor of a chaotic dynamical system from a sequence of measurements of one of its parameters taken at equal time intervals $\tau$ :

$$
\mathrm{Z}=\left\{z\left(t_{0}\right), z\left(t_{0}+\tau\right), \ldots, z\left(t_{0}+(m-1) \cdot \tau\right)\right\}
$$

This approach to the analysis of time series was mathematically substantiated in the work of F. Takens $[8,9]$. Reconstruction of the entire $d$-dimensional phase space (embedded space) from measurements of one variable is possible due to the fact that all variables of the state vector of the system are tied up in a general nonlinear process.

The maximum Lyapunov exponent is defined as:

$$
\lambda_{\max }=\lim _{t \rightarrow \infty} \lim _{\varepsilon \rightarrow 0} \frac{1}{t} \ln \left(\frac{\left|z(t)-z_{\varepsilon}(t)\right|}{\varepsilon}\right) .
$$

To determine the maximum Lyapunov exponent based on a finite series of measurements (4), we use the algorithm proposed by Rosentein [10]. Consider the representation of time series data as a trajectory in a reconstructed nested space. Individual trajectories of the system movement in the reconstructed space fluctuate along the main trend determined by the Lyapunov exponent spectrum. Then we can consider the distance $\Delta_{0}=\left|z\left(t_{0}\right)-z_{\varepsilon}\left(t_{0}\right)\right|$, as a deviation that should grow exponentially over time such that $\Delta_{t} \approx \Delta_{0} \varepsilon^{\lambda t}$. In this case, $\lambda$ will be equal to the maximum Lyapunov exponent. The spectrum of Lyapunov exponents is calculated as:

$$
\lambda_{\tau}(t)=\lim _{\varepsilon \rightarrow 0} \frac{1}{\tau} \ln \left(\frac{\left|z(t+\tau)-z_{\varepsilon}(t+\tau)\right|}{\varepsilon}\right) .
$$

If the spectrum $\lambda_{\tau}(t)$ shows a linear increase with the same slope for most of the trajectories, then this slope can be taken as an estimate of the maximum Lyapunov exponent $\lambda_{\max }$ (Fig. 6).

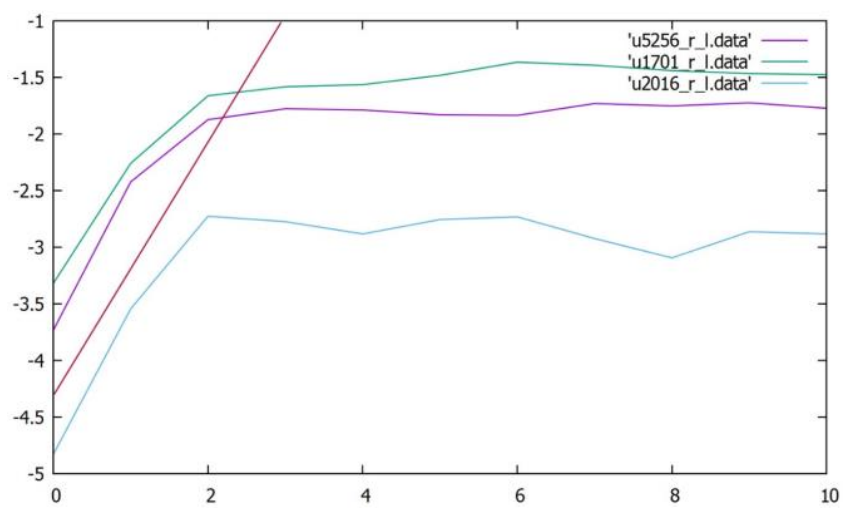

Fig. 6. Determination of the maximum Lyapunov exponent.

As the measurements by which the maximum Lyapunov exponent is determined, it is necessary to take the variable from the state vector of the system that is most influenced by other variables of the system. This 
will be the voltage value on the high-voltage buses remote from the buses, on which the voltage is maintained by the regulators.

To determine the Lyapunov exponents, the TISEAN library of nonlinear time series analysis was used [11].

The maximum Lyapunov exponent for the power system under consideration, determined from the power system state vector, is $\lambda_{\max }=2,238$. Thus, the chaotic behavior of the dynamic system is confirmed.

Given the chaotic behavior of the system, it is important to understand whether it is possible to predict the behavior of a chaotic system and what data set is needed to perform an adequate prediction. The rate of generation of new information in a number of measurements can be related to the rate of growth of distances in the space of measurements according to the work of Pesin [12]. The rate of information generation can be estimated by the value of the average mutual information:

$$
I_{A B}=\sum_{a_{i}, b_{i}} P_{A B}\left(a_{i}, b_{i}\right) \log _{2}\left(\frac{P_{A B}\left(a_{i}, b_{i}\right)}{P_{A}\left(a_{i}\right) P_{B}\left(b_{i}\right)}\right),
$$

where $a_{i}$ is an event from set $A, b_{i}$ is an event from set $B, P_{A}\left(a_{i}\right)$ is the probability of an event from the set $A$, $P_{B}\left(b_{i}\right)$ is the probability of an event from the set $B$, $P_{A B}\left(a_{i}, b_{i}\right)$ is the mutual probability of events.

If we take measurements $z(t)$ observed at times $t$ as the set of events $A$, and measurements $z(t+\tau)$ as events of the set $B$, then from the function of the average mutual information, we can determine the parameters of the series that are optimal for predictions measurements. So, to select the optimal discretization of measurements in [13], the first minimum of the function is found:

$$
\begin{gathered}
\mathrm{C}(\tau)=\sum_{t}|z(t)-\bar{z}||z(t+\tau)-\bar{z}|, \\
\bar{z}=\frac{1}{n} \sum_{t=1}^{n} z(t)
\end{gathered}
$$

With a predetermined measurement discreteness (as it is in the system under study), the size of the measurement vector $n$ used for forecasting can be varied:

$$
\min _{n} C(n)=\left\{\begin{array}{c}
\sum_{t}|z(t)-\bar{z}||z(t+\tau)-\bar{z}| \\
\bar{z}=\frac{1}{n} \sum_{t=1}^{n} z(t)
\end{array}\right.
$$

From the above calculations (Fig. 7), it can be seen that the first clear minimum appears after the 30th measurement snapshot, which, with a measurement frequency of 30 minutes, approaches the archive depth of one day. Thus, to obtain an adequate forecast in the model function, it is necessary to use more complex models than linear or moving average, which are often used in the Kalman filter.

As rightly noted in [14], the application of dynamic state estimation using the Kalman filter is limited by a slow change in the mode parameters and a forecasting horizon of up to $1 \mathrm{~min}$. Thus, the field of application of the dynamic state estimation proposed in [14] and similar works is limited to the automatic control of power plants, including for the purpose of emergency control.

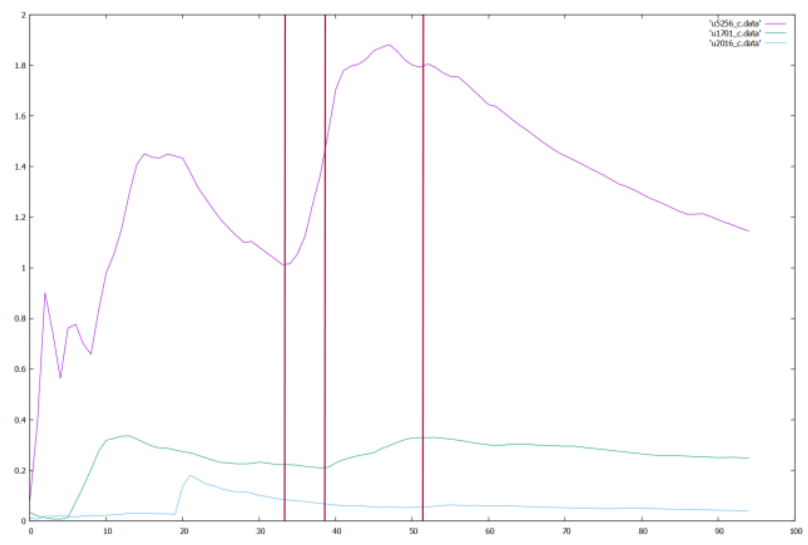

Fig. 7. Determination of the maximum Lyapunov exponent.

For the purposes of optimal control, a model is required that provides a forecast for a time of the order of a day. Such a model can be models based on artificial neural networks. Moreover, there are two options for using such models:

- direct use to obtain a forecast;

- use as a model of system behavior in dynamic state estimation using the Kalman filter.

The second variant of ANN application involves the use of a nonlinear Kalman filter, in particular, a sigmapoint filter.

\section{References}

1. A.Z. Gamm, I.N. Kolosok Detection of large errors in telemetry in electric power systems. Novosibirsk: Nauka, 2000. - 152 pp.

2. A.Z. Gamm Statistical methods for the state estimation of electric power systems. - Nauka, 1976.

3. R.E. Kalman A new approach to linear filtering and prediction problems //Journal of basic Engineering. 1960. - 82. - №. 1. - pp. 35-45.

4. S.S. Haykin Kalman filtering and neural networks. New York : Wiley, 2001. - pp. 221-269.

5. A.S. Debs, R.E. Larson A dynamic estimator for tracking the state of a power system //IEEE Transactions on Power Apparatus and Systems. 1970. - 7. - pp. 1670-1678.

6. H. Abarbanel Analysis of observed chaotic data. Springer Science \& Business Media, 2012.

7. A.Y. Loskutov Mathematical foundations of chaotic dynamical systems: a course of lectures //M.: MSU. -2008 .

8. F. Takens On the numerical determination of the dimension of an attractor//Dynamical systems and bifurcations. - Springer, Berlin, Heidelberg, 1985. pp. 99-106. 
9. L. Noakes The Takens embedding theorem //International Journal of Bifurcation and Chaos. 1991. - 1 - 04. - pp. 867-872.

10. M.T. Rosenstein, J.J. Collins, C.J. De Luca A practical method for calculating largest Lyapunov exponents from small data sets //Physica D: Nonlinear Phenomena. - 1993. - 65 - 1-2. - pp. 117134.

11. TISEAN Nonlinear Time Series Analysis,: https://www.pks.mpg.de/ tisean/ (Electronic resource)

12. Y.B. Pesin Lyapunov characteristic exponents and smooth ergodic theory // Uspekhi matematicheskikh nauk. - 1977. - 32. - №. 4 (196). - pp. 55-112.

13. Abarbanel H. Analysis of observed chaotic data. Springer Science \& Business Media, 2012.

14. Glazunova A. Dynamic state estimation //Monitoring, Control and Protection of Interconnected Power Systems. - Springer, Berlin, Heidelberg, 2014. - pp. 107-123. 